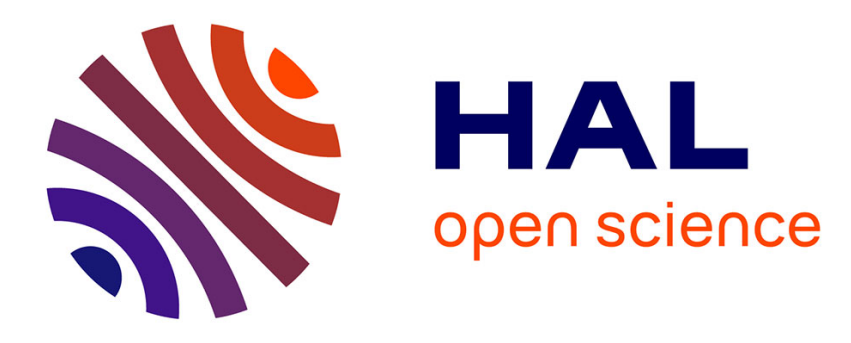

\title{
Diffusion of Hydrogen and Deuterium in Titanium Hydride and Deuteride
}

\author{
B. Kappesser, H. Wipf
}

\section{To cite this version:}

B. Kappesser, H. Wipf. Diffusion of Hydrogen and Deuterium in Titanium Hydride and Deuteride. Journal de Physique IV Proceedings, 1996, 06 (C8), pp.C8-73-C8-76. 10.1051/jp4:1996813 . jpa00254561

\section{HAL Id: jpa-00254561 https://hal.science/jpa-00254561}

Submitted on 1 Jan 1996

HAL is a multi-disciplinary open access archive for the deposit and dissemination of scientific research documents, whether they are published or not. The documents may come from teaching and research institutions in France or abroad, or from public or private research centers.
L'archive ouverte pluridisciplinaire HAL, est destinée au dépôt et à la diffusion de documents scientifiques de niveau recherche, publiés ou non, émanant des établissements d'enseignement et de recherche français ou étrangers, des laboratoires publics ou privés. 


\title{
Diffusion of Hydrogen and Deuterium in Titanium Hydride and Deuteride
}

\author{
B. Kappesser and H. Wipf \\ Institut für Festkörperphysik, Technische Hochschule Darmstadt, Hochschulstrasse 6, 64289 Darmstadt, \\ Germany
}

\begin{abstract}
In the hydrogen $(\mathrm{H})$ concentration range $1.6 \leq x \leq 1.8, \mathrm{TiH}_{x}$ exists in a $\delta$ phase where $\mathrm{Ti}$ forms a fcc lattice in which the $\mathrm{H}$ occupies tetrahedral interstitial sites. We studied the diffusion of the $\mathrm{H}$ in this phase by mechanical spectroscopy (temperatures from 5 to $370 \mathrm{~K}$, vibrating reed technique, frequencies from 169 to $1290 \mathrm{~Hz}$ ). We find a large H-induced Zener-relaxation peak between 240 and $270 \mathrm{~K}$ (for the present frequencies) from which we determined the jump rate of the $\mathrm{H}$ interstitials with the help of a recent theoretical model for the Zener relaxation. The jump rate follows an Arrhenius relation with an activation enthalpy of $(0.49 \pm 0.04) \mathrm{eV}$. An extrapolation of the present results agrees well with previous high-temperature data from neutron spectroscopy and nuclear magnetic resonance.

For concentrations $x>1.8$, a continuous (ferroelastic) phase transition takes place below $\sim 320 \mathrm{~K}$, changing the fcc $\mathrm{Ti}$ lattice into fct ( $\epsilon$ phase). The phase transition is accompanied by a drastic softening of Young's modulus which allows a very precise determination of the transition temperature.
\end{abstract}

\section{INTRODUCTION}

At high hydrogen $(\mathrm{H})$ concentrations $(1.6 \leq x \leq 2.0)$, the system $\mathrm{TiH}_{x}$ exists in a $\delta$ or in a $\epsilon$ phase (for $x>1.8$ and below $\sim 320 \mathrm{~K}$ ) [1]. The Ti atoms form a fcc ( $\delta$ phase) or a fct ( $\epsilon$ phase) lattice in which the $\mathrm{H}$ occupies tetrahedral interstitial sites [1]. In the $\delta$ phase, these tetrahedral sites form a sc sublattice which is (slightly) tetragonally distorted in the $\epsilon$ phase. Since there are two tetrahedral sites per Ti atom, all tetrahedral sites are occupied for $x=2$.

In this paper, we report the results of a mechanical-spectroscopy study in which we investigated (i) the diffusion of the $\mathrm{H}$ interstitials in the $\delta$ phase of $\mathrm{TiH}_{x}$ and (ii) the $\delta$-to- $\epsilon$ phase transition. In the $\delta$ phase $(x<1.8)$, we find a strong H-induced anelastic relaxation peak (at $\sim 240 \mathrm{~K}$ for a frequency of $169 \mathrm{~Hz}$ ) which is identified as a Zener relaxation of the $\mathrm{H}$ interstitials. We determine the jump rates of the $H$ with the help of a recent theoretical model for the Zener relaxation [7]. An extrapolation of the jump rates towards higher temperatures agrees well with previous quasielastic neutron scattering (QNS) [2] and nuclear magnetic resonance (NMR) [3-6] data, measured between 400 and $1200 \mathrm{~K}$.

The ferroelastic (continuous) $\delta$-to- $\epsilon$ phase transition (occurring for $x>1.8$ ) is accompanied by a drastic softening of Young's modulus in $\langle 100\rangle$ direction. This fact allows a reliable determination of the transition temperatures from our experiments.

\section{SAMPLE PREPARATION AND EXPERIMENTAL DETAILS}

The measurements were carried out on polycrystalline $\mathrm{TiH}_{x}$ samples $(\sim 50 \mathrm{~mm}$ long, $5 \mathrm{~mm}$ wide and $0.1 \mathrm{~mm}$ thick). The samples were prepared from Ti foils (Goodfellow, nominal purity 99.99\%) doped with $\mathrm{H}$ in an UHV-system by adding appropriate amounts of $\mathrm{H}_{2}$ gas $\left(\mathrm{H}_{2}\right.$ pressures up to 1.5 bar, temperatures between 300 and $700{ }^{\circ} \mathrm{C}$ ). During $\mathrm{H}$ doping, the $\mathrm{H}_{2}$ gas pressures were varied extremely slowly to avoid crack formation due to inhomogeneous $\mathrm{H}$ distributions. The resulting $\mathrm{H}$ concentrations were determined (i) from the increase of the sample mass and (ii) by vacuum 
extraction after the measurements were completed (experimental accuracy $\pm 1 \%$ ).

The anelasticity measurements were performed in a temperature-controlled vacuum chamber with the help of a vibrating reed apparatus (electrostatic drive, FM detection). During the measurements, the sample was clamped at different positions along its long side which allowed a change of the vibrational frequencies between 169 and $1290 \mathrm{~Hz}$. The mechanical energy dissipation (internal friction) $Q^{-1}$ was measured in both heating and cooling cycles in the temperature range between 4 and $370 \mathrm{~K}$ (heating and cooling rates $\leq 15 \mathrm{~K} / \mathrm{h}$ ).

\section{EXPERIMENTAL RESULTS AND DISCUSSION}

\subsection{H Diffusion in the $\delta$ Phase}

Figure 1 presents $Q^{-1}$ spectra of a $\mathrm{TiH}_{1.66}$ sample taken at three different frequencies. Between 240 and $270 \mathrm{~K}$, the spectra demonstrate a large $\mathrm{H}$-induced relaxation peak that shifts to higher temperatures with increasing frequency, thus indicating a thermally activated relaxation process. From the temperature-versus-frequency shift, the activation enthalpy of the relaxation process is found to be $(0.49 \pm 0.04) \mathrm{eV}$. For this activation enthalpy, the widths of the relaxation peaks exceed those of an ideal Debye relaxation by more than $70 \%$. Therefore, the relaxation process is controlled by a spectrum of relaxation times.

We consider the microscopic mechanism of the relaxation process now. In the $\delta$ phase, with its sc tetrahedral $\mathrm{H}$ sublattice, a simple Snoek-type $\mathrm{H}$ relaxation process between differently oriented $\mathrm{H}$ sites can be excluded for symmetry reasons [8]. Accordingly, we conclude that the observed relaxation is caused by a Zener relaxation of the $\mathrm{H}$ interstitials [8]. The Zener relaxation is an anelastic relaxation process due to stress-induced changes of the atomic order, which means, in the present case, due to stress-induced changes of the distribution of the $\mathrm{H}$ interstitials on their tetrahedral sites.

$\mathrm{H}$ interstitials in metals are the realization of a diffusing lattice gas. The host-lattice metal atoms can be assumed to be immobile whereas the $H$ atoms can diffuse on their interstitial sublattice. The anelastic Zener relaxation of such a diffusing lattice gas was considered in a recent model calculation [7]. The model assumes a lattice gas that is non-interacting except for the fact that a given lattice site can be occupied only once. It holds for a sc lattice (in agreement with the sc $\mathrm{H}$ sublattice of $\delta$-phase $\mathrm{TiH}_{x}$ ), and it considers lattice gas atom (i.e. a $H$ interstitial) that jump with a jump rate $\Gamma$ to an unoccupied nearest-neighbour site. The anelastic relaxation results from a stress-induced formation and dissolution of bonds between (paired) lattice gas atoms that occupy nearest-neighbour sites. For these model assumptions, the relaxation spectra of both the compressibility $3 \cdot\left(s_{11}+2 s_{12}\right)$ and the shear compliance $2 \cdot\left(s_{11}-s_{12}\right)$ were calculated rigorously correctly $\left(s_{11}, s_{12}\right.$ and $s_{44}$ are elastic compliances in Voigt's notation [11], where $s_{44}$ does not relax for symmetry reasons). The relaxation spectra exhibit peaks with a maximum for a frequency $f=1.92 \cdot \Gamma$ and $f=1.85 \cdot \Gamma$ in the case of the compressibility and the shear compliance $2 \cdot\left(s_{11}-s_{12}\right)$, respectively. Further, both relaxation spectra are found considerably broadened in comparison with a Debye relaxation, which shows the presence of a spectrum of relaxation times.

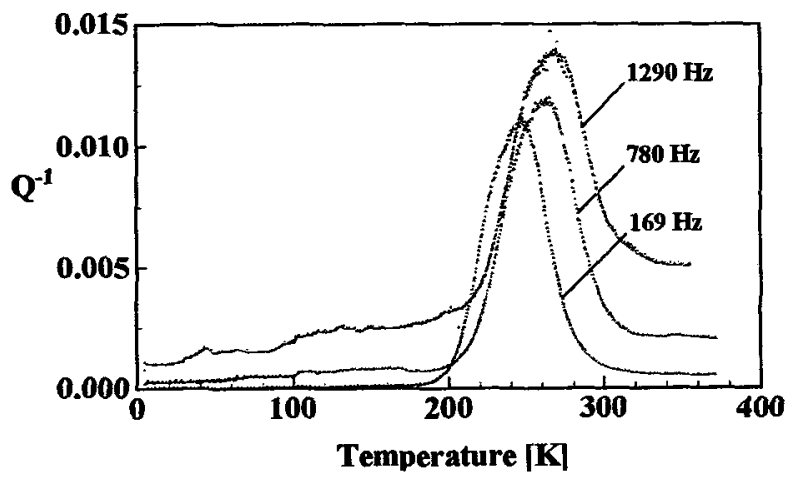

Figure 1: Energy dissipation $Q^{-1}$ of a $T_{i} H_{1.66}$ sample as a function of temperature. The indicated frequencies hold for the maximum of the relaxation peaks. 


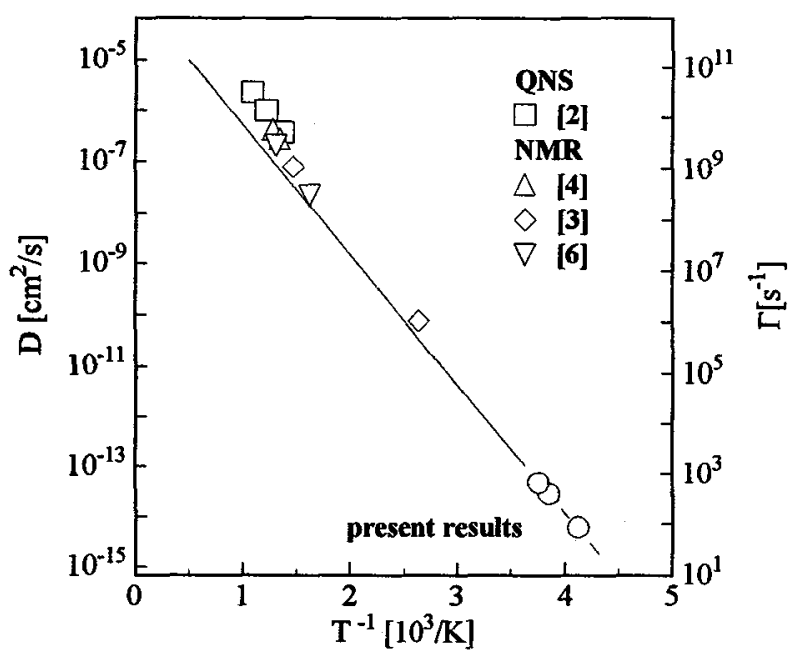

Figure 2: Jump rate $\Gamma$ (right hand scale) and diffusion coefficient $D$ (left hand scale) of the $H$ atoms as a function of reciprocal temperature $T^{-1}$. The present data are shown together with previous ones determined by QNS and

NMR. The straight line indicates an extrapolation of the present results to higher temperatures.

This agrees with our present observation. We point out that a relaxation spectrum is found although all the lattice gas atoms (or $\mathrm{H}$ interstitials) are assumed to have a single jump rate $\Gamma$ to an unoccupied nearest neighbour site. The reason for this is that the formation and dissolution of the bonds between the lattice gas atoms involve in part also longer diffusion processes.

At the relaxation peak maxima, the relations between $f$ and $\Gamma$ are nearly identical for both the compressibility and the shear compliance $2 \cdot\left(s_{11}-s_{12}\right)$ (i. e. $f \simeq 1.9 \cdot \Gamma$ ). Therefore, the jump rate $\Gamma$ can be determined from the relaxation peak maxima of our present experiments on polycrystalline samples since we need not know to what extent the compressibility and the compliance $2 \cdot\left(s_{11}-s_{12}\right)$ contribute to the measured relaxation. Figure 2 presents our results for $\Gamma$ (right hand scale) in an Arrhenius presentation, together with results for $\Gamma$ and the self diffusion coefficient $D$ (left hand scale) as derived from previous QNS [2] and NMR [3-6] experiments. The relation between $D$ and $T$ that was used in calculating the data in Fig. 2 is given in [2].

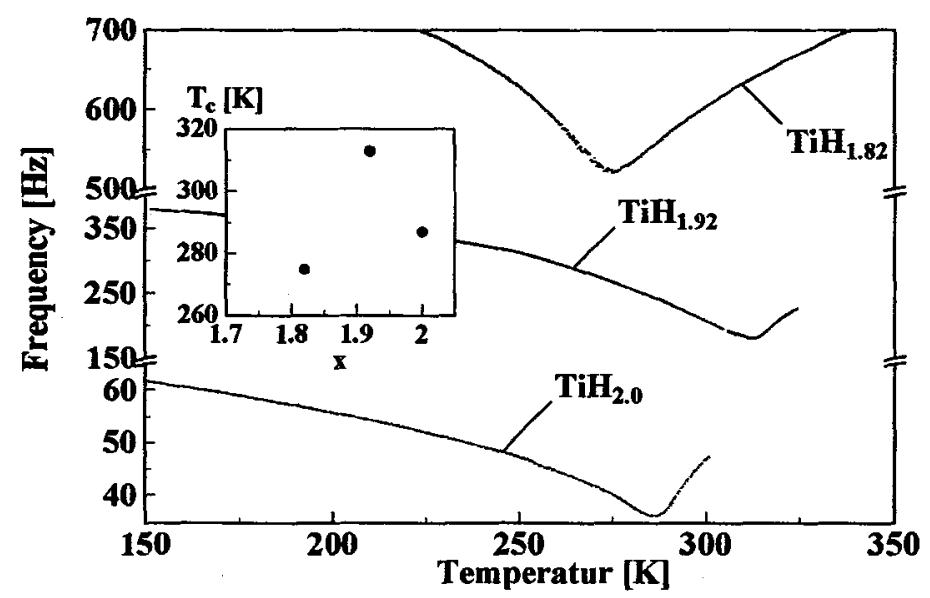

Figure 3: Temperature dependence of the vibrational frequency of samples with different $H$ concentrations as a function of temperature. The insert shows the results for the temperature $T_{c}$ of the phase transition as a function of the $H$ concentration $x$. 
The straight line in Fig. 2 shows an extrapolation of the present mechanical-spectroscopy results towards higher temperatures (with an activation enthalpy $0.49 \mathrm{eV}$ ). It is seen that this extrapolation agrees very well with the QNS and NMR data, in particular under consideration of the fact that the extrapolation extends over nine decades.

\subsection{The Ferroelastic $\delta$-to- $\epsilon$ Phase Transition}

We discuss now the (continuous) ferroelastic phase transition between the high-temperature $\delta$ phase (fcc $\mathrm{Ti}$ lattice) and the $\epsilon$ phase (fct), taking place for $x \geq 1.8$ for temperatures below $\sim 320 \mathrm{~K}$. Figure 3 shows, for samples with three different $\mathrm{H}$ concentrations, the measured vibrational frequencies as a function of temperature. The data exhibit a clearly identifiable frequency minimum at the transition temperature $T_{c}$, caused by the drastic softening of Young's modulus in $\langle 100\rangle$ direction. The frequency minimum allows a very precise determination of $T_{c}$, more precise, for instance, than by $X$-ray or neutron diffraction [1]. The precision in our determination of $T_{c}$ is also expressed by the fact that identical $T_{c}$ values were measured completely independently of (i) the vibrational frequency and (ii) whether the data were taken in heating or cooling cycles. The insert in Fig. 3 shows a compilation of our present results for $T_{c}$ as a function of $\mathrm{H}$ concentration $x$. The results agree essentially with previous data, although they show clearly a maximum of $T_{\mathfrak{c}}$ which is not reported so far [1].

\section{CONCLUSION}

$\delta$-phase $\mathrm{TiH}_{x}$ exhibits a large anelastic relaxation peak caused by a Zener relaxation of the $\mathrm{H}$ interstitials. With the help of a recent model calculation for the Zener relaxation [7], we determined the jump rates of the $\mathrm{H}$ interstitials in $\delta$-phase $\mathrm{TiH}_{x}$ from our measured relaxation peaks. The temperature dependence of the jump rate follows an Arrhenius relation with an activation enthalpy of $(0.49 \pm 0.04) \mathrm{eV}$. An extrapolation of the measured jump rates towards higher temperatures agrees well with previous NMR and QNS data.

The softening of Young's modulus at the ferroelastic (continuous) $\delta$-to- $\epsilon$ phase transition allows a precise determination of the transition temperature. This temperature exhibits a maximum around a $\mathrm{H}$ concentration $x=1.92$.

\section{References}

[1] A. San-Martin, F. D. Manchester, Bull. Alloy Phase Diagrams 8, 30 (1987).

[2] U. Stuhr, D. Steinbinder, H. Wipf, B. Frick, Europhys. Lett. 20, 117 (1992).

[3] C. Korn, D. Zamir, J. Phys. Chem. Solids, Vol. 31, 489-502 (1970).

[4] L. D. Bustard, R. M. Cotts, E. F. W. Seymour, Phys. Rev. B 22, 12 (1980).

[5] C. Korn, S. D. Goren, Phys. Rev. B 22, 10 (1980).

[6] U. Kaess et al., to be published.

[7] H. Wipf, B. Kappesser, J. Phys.: Condens. Matter, in print.

[8] A. S. Nowick, B. S. Berry, Anelastic Relaxation in Solids, Academic Press, New York, 1972. 